\section{IQ and vitamin supplements}

SIR - None of Steven Blinkhorn's criticisms (Nature 350, 13; 1991) of the study of IQ improvement following vitamin and mineral supplementation ${ }^{1}$, financed by the Dietary Research Foundation, are acceptable. His chief criticism is directed at the statistical treatment. He argues that significance tests should have been carried out on all the tests and supplementation formulae issued. But this idea ignores the fact that we tested specific hypotheses, rather than proceeding blindly. We hypothesized, on the basis of past findings and psychological theory, that tests of fluid ability (nonverbal tests) would show an effect, while tests of crystallized ability (verbal tests) would not. We made the Wechsler (WISC-R) individually administered test our main tool, as any psychologist would do; the data bore out both hypotheses. It would have been quite nonsensical to count the failure of the verbal tests against our hypothesis; this is exactly what was expected.

We also included some tests of scholastic achievement, which were in any case administered to the children not as tests of our hypothesis but because of the possible interest of any findings. (Our hypothesis would predict no effect for such a verbal test.) To have included the results (which suggest a mildly positive effect) in support of our theory would have been quite inappropriate.

We also had the chance to administer the Raven Matrices test, but only once, so that there is no test-retest comparison; it was also administered after 4 weeks of supplementation, rather than the 12 weeks in our main study. The insignificant overall superiority of the supplementation groups replicates the finding by Nelson et $\mathrm{al}^{2}$ that short periods of

\section{Due credit}

SIR - I was glad to read Steven Dickman's favourable report (Nature 350, 179; 1991) on the European Research Conferences organized under the auspices of the European Science Foundation and to be funded, it is hoped, through the European Communities. I should, however, point out that even though I strongly back this promising scheme to bring the most talented young European researchers together in the formative period of their scientific careers, the men who deserve most of the credit for having established this programme are Professor Heinz Georg Wagner, Universität Göttingen, who began to work for this goal more than two years ago when he was a vice president of the Deutsche Forschungsgemeinschaft, and Professor Claude Fréjacques, vice president of the European Science Foundation.

Hubert Mark

(President)

Deutsche Forschungsgemeinschaft, D-5300 Bonn 2, Germany supplementation are insufficient to produce marked changes; this too is not relevant to our main hypothesis, and cannot be used to disprove it. But even here, for reasons given in our article, the largest dose does give a significant degree of superiority over the control group.

Finally, the MAT group test showed the same overall pattern of results (best for 100 per cent of the Recommended Daily Allowance, worst for the placebo) on the WISC-R, but the large standard deviation that we found suggests that the statistical power of the test is insufficient, perhaps because of the children's lack of interest in the test often observed in group testing. This suggests that for replication of future studies, individual tests might be preferable.

Yet Blinkhorn suggests that, to prove our case, we used "several forms of analysis chosen to suit a preferred hypothesis as proof of that hypothesis"; this is difficult to understand. We used the same method of analysis for the MAT and for the WISC-R data, and reported nonsignificance. To find a result nonsignificant does not seem to suggest that we picked arbitrarily a type of analysis which would make the results appear significant. To call this "a blatant piece of post-hoccery" seems hardly the measured kind of comment one expects from a fellow scientist.

Blinkhorn argues that, because the results of the blood sample analysis were not yet completed, we should have delayed publication. But the blood analysis data relate to a different problem from that treated in our report, that of which vitamin or minerals are responsible for the effect established in our study. Similarly, the data from our British study are a replication of our US study; they will strengthen the conclusions, but we believe that the published data are sufficient to establish the conclusions.

Some of the demands for further information by Blinkhorn and others are being met by S. J. Schoenthaler ${ }^{3}$, but one point may need comment. Blinkhorn asks: "What of those children who did worse than expected in the treatment group?" It is difficult to understand the question, but the implication that the treatment might have lowered their IQ is breathtakingly naive. Obviously there will be a chance distribution about the mean, so that some do better, others worse than the average. There is no hint in the data of any significant deterioration of IQ in any of the supplementation children; Blinkhorn is welcome to re-analyse our data to satisfy himself on that point.

Blinkhorn finally criticized me for saying that the best policy would be to administer a 100 per cent RDA dose to all children. He leaves out the context, which makes it plain that only a minority would benefit, that such a minority could perhaps be identified by blood sample analysis, but that, in the absence of such analysis, general supple- mentations might be the best policy. He also overlooks our repeated statement that improving a child's diet would be the most useful way of improving his or her supply of vitamins and minerals.

These are only some of the inaccuracies and errors in Blinkhorn's review; readers are invited to read our symposium to make up their own minds.

Institute of Psychiatry,

H. J. EYSENCK

De Crespigny Park

Denmark Hill, London SE5 8AF

1. Schoenthaler, S. J., Amos, S. P., Eysenck, H. J., Peritz, E. \& Yudkin, I Personality and Individual Differences 12 351-362 (1991).

2. Nelson, M., Naismith, D. J., Burley, V., Gatenby, S. \& Geddes, N. Br. J. Nutr. 64, 13-22 (1990)

Schoenthaler, S. J. Nature (in the press).

\section{Scientific licence}

SIR - In his review of Frank Close's book Too Hot to Handle: The Race for Cold Fusion (Nature 350, 29; 1991), Sir Brian Pippard ends: "The book should be read as an exemplary tale by all who are concerned about the conflicting demands of scientific integrity, personal ambition and public interest." Other professional groups have faced such conflicting demands by laying down enforceable codes of conduct for their members, for example the medical profession. I suggest the following scheme will go a fair way towards balancing Pippard's conflicting demands.

(1) Scientists must be licensed to practice, on the lines of medical practitioners, by professional watch-dog bodies. Scientists against whom cases of scientific malpractice and/or fraud have been proved should have their licences withdrawn for varying periods of time [depending on the severity of the malpractice] including, in extreme cases, permanent cancellation. No doubt a malpractice insurance market for scientists will develop - no harm in that.

(2) Analogous to the 'polluter pays' principle, scientists who wilfully pollute the scientific environment should be made to pay the costs of 'clean-up', that is, the costs of all the cross-checking, experimentation, conferences and theoretical wild-goose chases that other scientists are induced to engage in, only to discover a near-hoax. Only a fraction of the costs of such clean-up can be borne by the 'polluting' scientists themselves; the major costs will have to be borne by public- and private-sector employers, who will therefore have a strong incentive to enforce high standards of integrity and validation on the scientific output of their employees.

Secretariat of SA to RM,

V. SIDDHARTHA

Ministry of Defence,

Room No. 131, ' $B$ ' Wing,

Sena Bhavan, DHQ PO,

New Delhi 110 011, India 